\title{
NO-HYPE: a novel hydrodynamic phantom for the evaluation of MRI flow measurements
}

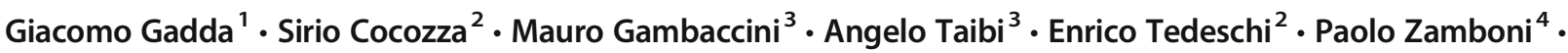 \\ Giuseppe Palma ${ }^{5}$
}

Received: 13 November 2020 / Accepted: 7 June 2021 / Published online: 8 August 2021

(C) The Author(s) 2021

\begin{abstract}
Accurate and reproducible measurement of blood flow profile is very important in many clinical investigations for diagnosing cardiovascular disorders. Given that many factors could affect human circulation, and several parameters must be set to properly evaluate blood flows with phase-contrast techniques, we developed an MRI-compatible hydrodynamic phantom to simulate different physiological blood flows. The phantom included a programmable hydraulic pump connected to a series of pipes immersed in a solution mimicking human soft tissues, with a blood-mimicking fluid flowing in the pipes. The pump is able to shape and control the flow by driving a piston through a dedicated software. Periodic waveforms are used as input to the pump to move the fluid into the pipes, with synchronization of the MRI sequences to the flow waveforms. A dedicated software is used to extract and analyze flow data from magnitude and phase images. The match between the nominal and the measured flows was assessed, and the scope of phantom variables useful for a reliable calibration of an MRI system was accordingly defined. Results showed that the NO-HYPE phantom is a valuable tool for the assessment of MRI scanners and sequence design for the MR evaluation of blood flows.
\end{abstract}

Keywords Phase-contrast MRI $\cdot$ Human circulation $\cdot$ Blood flow simulation $\cdot$ Hydrodynamic phantom

\section{Introduction}

Quality control with a quantitative magnetic resonance imaging (MRI) phantom is necessary to ensure the accuracy and precision of results [1]. Indeed, system constancy data should be tracked regularly at all MRI systems and especially those used for

Angelo Taibi

angelo.taibi@unife.it

1 Section of Ferrara, National Institute for Nuclear Physics (INFN), 44122 Ferrara, Italy

2 Department of Advanced Biomedical Sciences, University of Naples "Federico II", 80131 Napoli, Italy

3 Department of Physics and Earth Sciences, University of Ferrara, 44122 Ferrara, Italy

4 Vascular Diseases Center - Translational Surgery Unit, University of Ferrara, 44124 Ferrara, Italy

5 Institute of Biostructures and Bioimaging, National Research Council, 80145 Napoli, Italy quantitative measurements [2]. Measurement of blood flow velocity using phase-contrast (PC) MRI technique has been performed since the advent of MRI in the early 1980 [3], thus demonstrating a valuable non-invasive technique $[4,5]$ for both qualitative and quantitative assessment of flow [6-8].

Currently, the technique is benefiting from the incorporation of several technological advancements in MRI, such as increased gradient and field strengths, and higher signal-tonoise ratio (SNR) from multichannel coils. Also, timeresolved measurement of the blood flow velocity using cardiac-gated PC-MRI is gaining increasing interest in the clinical practice due to its capability to characterize the entire hemodynamic cycle [9]. Such technological improvements led in turn to the introduction of novel methods of data acquisition and analysis, which allow to derive additional information such as flow rate [10], pressure [11], and wall shear stress (WSS) [12].

The hemodynamic information from rapid PC-MRI scans is valuable in different clinical scenarios [13], but the accuracy of PC-MRI measurements is often unknown, thus limiting its actual incorporation in clinical practice. PC-MRI has been 
extensively studied in vivo in the aortic arch and carotid arteries [14-16], as well as in small structures such as cerebral arteries and intracranial aneurysms [17]. However, any accurate in vivo validation of velocity measurement is challenging, and an assessment of the technique through a dedicated phantom should be warranted beforehand, as exhaustively stated in the paper by Keenan et al. [2]. Such work reports that the Radiological Society of North America (RSNA), through the Quantitative Imaging Biomarkers Alliance (QIBA), promotes the development of quantitative imaging phantoms [18]. Efforts are also made by other institutions such as the American Association of Physicists in Medicine and the European Communities Biomedical Engineering Advisory Committee [19-22].

Given this background, in this study, we built and validated a flow phantom to understand, test, and optimize PC-MRI measurements for reliable clinical settings. The main contributions of the study are:

To develop a MRI-compatible hydrodynamic phantom to simulate physiological blood flows

To build the phantom and check the mechanical and electrical robustness of the pumping system

To test the phantom in a clinical setup for the first time

To collect and analyze the data acquired from the MRI scanner

To highlight the aforementioned contributions, the Methods section is divided in subsections describing:

The hydraulic pump for blood flow simulation

The design of the hydrodynamic phantom

The flow data acquisition and analysis

The Results section is divided in subsections that report and analyze simulations of:

Constant flow

Pulsatile flow:

- Sine flow

- Physiologic flow

\section{Methods}

\subsection{Hydraulic pump for blood flow simulation}

The CompuFlow 1000 MR (Shelley Medical Imaging Technologies) [23] is a positive displacement pump designed for researches on simulated blood flow [24]. It is a two unit system consisting of a control assembly unit and a pump assembly unit (Fig. 1, left) that allows to dispense fluid at precise and accurate steady and pulsatile flow rates.
The piston is driven on a precise lead screw by a motion controller, programmed to eject the appropriate volume of fluid at a predetermined time interval. The resulting flow is accurate within $\pm 1 \%$ over a range of $0.1-35 \mathrm{ml} / \mathrm{s}$. By interchanging the outlet and inlet paths when the piston reaches the end of its travel, a nearly uninterrupted output flow is achieved, thanks to a 4-port directional flow control valve; the pump refills one side of the cylinder, meanwhile it pumps fluid out the other side. A dedicated software allows to run the pump and perform both constant and pulsatile flow rates of the desired waveform. Such flow waveforms are synchronized with the MRI sequences through a custom, in-house developed, pulse oximeter device simulating the heartbeat, which is used as input to the MRI scanner for the retrospective gating of the signal acquisitions. The designed waveforms are provided as a file that contains the data points sampling the waveform (normalized between -1 and +1 ), the scale value that defines the peak value, and the time interval between the points. As the pump is not MR-compatible, it must be placed outside the MRI room.

\subsection{Design of the hydrodynamic phantom}

The MR-compatible phantom was connected to the pump outside the MRI room through long polyvinyl chloride (PVC) connections, which also ensure laminar flow and avoid turbulence. The phantom is made of 12 straight and parallel glass pipes of variable diameters (ranging from $(9.0 \pm 0.1) \mathrm{mm}$ to $(15.0 \pm 0.1) \mathrm{mm})$ located into a bicylindrical plexiglass case (Fig. 1, right).

The length of each glass pipe, as well as the distance between hydraulic connectors, is $1000 \pm 1 \mathrm{~mm}$. The lengths of the two plexiglass cylinders are $460 \pm 1 \mathrm{~mm}$ and $440 \pm 1 \mathrm{~mm}$, while the diameters are $196 \pm 1 \mathrm{~mm}$ and $146 \pm 1 \mathrm{~mm}$, respectively. The two cylinders are coaxial and fixed together at the bases to form the protective case for the glass pipes. In the central part of the case, an isolated chamber (length $(440 \pm 1) \mathrm{mm}$ ) contains the soft tissue-mimicking liquid crossed by the glass pipes. A large number of tissue-mimicking materials are described in the literature, created using different techniques [25-29]. The material used in this experiment is a water solution of $10 \mathrm{mmol}$ per liter of $\mathrm{CuSO}_{4}$ [30]. The ion concentration of $\mathrm{CuSO}_{4}$ in water leads to relaxation time values similar to the ones of human soft tissues at clinical magnetic field intensities. Such mixture is cheap and easy to be produced in-house, thus allowing to perform several tests at different $\mathrm{CuSO}_{4}$ concentration before filling the phantom. We are aware that solutions can change its properties in time. For example, its homogeneity could change, thus compromising the tissue-mimicking property with respect to MRI. We performed the presented and other acquisitions in several weeks, and we never 

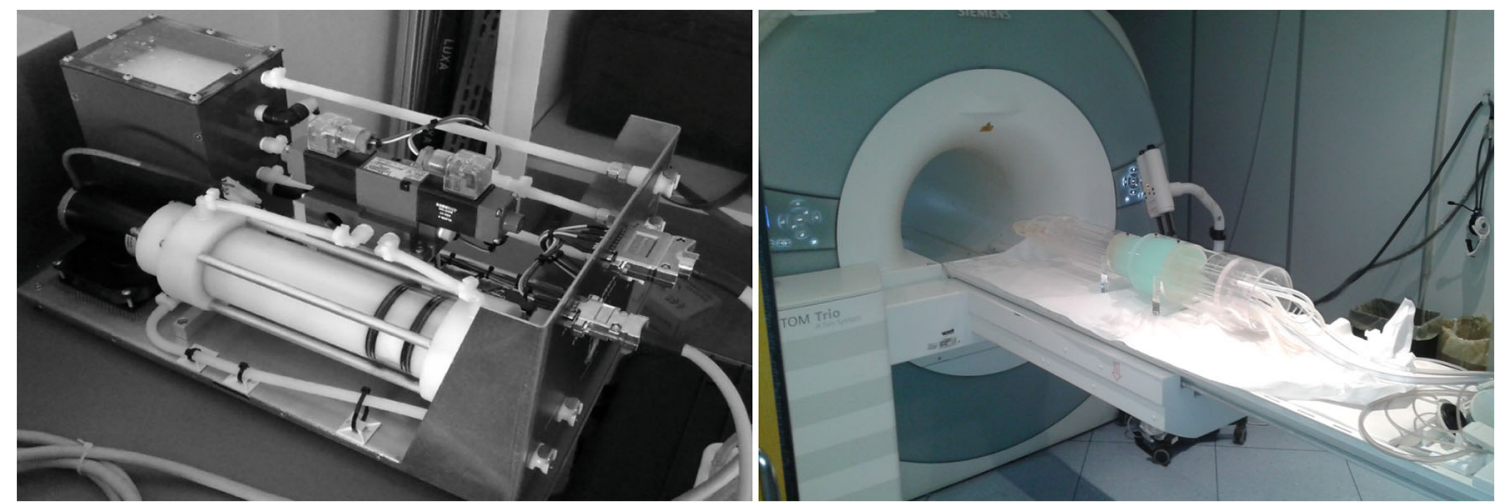

Fig. 1 Left: CompuFlow 1000 MR (internal of the pump unit). Right: setting of the hydrodynamic phantom before a MRI acquisition session. Soft tissuemimicking material is hosted in the central part of the phantom (light blue chamber). Glass pipes pass through the chamber carrying the blood mimicking fluid

observed any significant change in the MR image quality. Therefore, we can state that the solution is stable enough to allow reliable measurements in different acquisition sessions. The blood-mimicking fluid (BMF) was produced by Shelley Medical Imaging Technologies and simulates the physical characteristics of blood for MRI studies (see Table 1) [31].

\subsection{Flow data acquisition and analysis}

The acquisitions were performed on a $3 \mathrm{~T}$ scanner (Siemens Trio, Siemens Medical Systems, Erlangen, Germany). The pumping system was set to produce both constant and pulsatile flows, with amplitudes and frequencies selected to span a range of physiological interest (the complete list of the acquisition schemes is reported in Table 2).

Each 2D acquisition was set up on the same axial slice and produced a complex dataset (magnitude-and-phase representation, see Fig. 2) composed of 30 frames sampling the waveform period; as such, the temporal resolution depended on the period of the incoming pulsation.

Flow values are estimated from the phase images, whereas the magnitude images are used for anatomical reference to properly assess the pipe contours through regions of interest (ROIs). All the pipes appear intense in magnitude images, while they appear bright or dark in phase images, depending on the flow direction. Indeed, the phase shift accumulated at the echo time $T_{E}$ by a fluid element whose position $x(t)$ changes according to:

$x(t)=\sum_{n=0}^{+\infty} \frac{d^{n} x}{d t^{n}}(0) \frac{t^{n}}{n !}$

under the action of an arbitrary flow-encoding gradient pulse $\mathbf{G}(\mathrm{t})$ is given by: $\phi=\gamma \int_{0}^{T_{E}} \mathbf{G}(t) \cdot x(t) d t=\gamma \sum_{n=0}^{+\infty} \frac{\mathbf{m}_{\mathrm{n}}}{n !} \cdot \frac{d^{n} x}{d t^{n}}(0)$

where $\gamma$ is the gyromagnetic ratio of the imaged nuclear species $\left({ }^{1} \mathrm{H}\right.$, in this case) and $\mathbf{m}_{\mathrm{n}}$ represents the $n$-th moment of the gradient pulse:

$\mathbf{m}_{\mathrm{n}}=\int_{0}^{T_{E}} \mathbf{G}(t) t^{n} d t$

If we consider a steady flow:

$\phi=\phi_{0}+\gamma \mathbf{m}_{1} \cdot \mathbf{v}_{\mathbf{0}}$

where $\mathbf{v}_{\mathbf{0}}$ is the flow velocity and $\phi_{0}$ accounts for a variety of phenomena not related to the flow. However, if a second acquisition with reversed gradient $-\vec{G}(t)$ is collected, the phase difference between the two images is given by:

$\Delta \phi=2 \gamma \mathbf{v}_{0} \cdot \mathbf{m}_{1}$

Therefore, it is possible to derive the velocity component parallel to $\mathbf{m}_{\mathbf{1}}$ as:

$v_{0}=\frac{\Delta \phi}{2 \gamma\left|\mathbf{m}_{1}\right|}$

It also follows from Eq. (6) that aliasing occurs when the speed exceeds the velocity-encoding (VENC) parameter, which is defined as:

Table 1 MRI properties of the used BMF. The manufacturer provides information about $1.5 \mathrm{~T}$ magnetic field only [31]

\begin{tabular}{ll}
\hline $\mathrm{T}_{1}$ & $850 \mathrm{~ms}$ \\
$\mathrm{~T}_{2}$ & $170 \mathrm{~ms}$ \\
Density & $1.02 \mathrm{~g} / \mathrm{cm}^{3}$ \\
Viscosity & $4.1 \mathrm{mPa} \cdot \mathrm{s}$ \\
\hline
\end{tabular}


Table 2 List of acquisitions performed

\begin{tabular}{llll}
\hline Flow type & $\mathrm{Q}[\mathrm{ml} / \mathrm{s}]$ & VENC $[\mathrm{cm} / \mathrm{s}]$ & $\mathrm{f}[\mathrm{Hz}$ \\
\hline Constant & 5 & 50 & $/$ \\
& 5 & 75 & $/$ \\
& 10 & 50 & $/$ \\
Sine & 10 & 75 & $/$ \\
& 5 & 50 & 1 \\
& 10 & 50 & 1 \\
& 15 & 50 & 1 \\
& 10 & 50 & 0.3 \\
& 10 & 50 & 0.5 \\
& 10 & 50 & 0.75 \\
& 10 & 50 & 1.5 \\
& 10 & 50 & 2 \\
& 10 & 50 & 3 \\
\hline
\end{tabular}

$\mathrm{VENC}=\frac{\pi}{2 \gamma\left|\mathbf{m}_{1}\right|}$

A complete list of symbols used in the above equations is reported in Table 3 .

We used the software package SPIN 1.5.7 (SpinTech, Inc., Bingham Farms, MI, USA) [32] to extract flow data from DICOM images. SPIN is an advanced image viewing and quantitative MR post-processing software that has been created to provide the radiologist with a tool for quantitative image analysis. Beside iron content, cerebral microbleeds, white matter hyperintensities, and perfusion weighted imaging, SPIN is particularly useful for the detection and analysis of blood flow through dedicated plugins.

The pipes were contoured on a given acquisition through an automated vessel boundary detection, which is based on region growing method with full-width half-maximum thresholding, so as to allow for consistency in inter- and intra-processing reliability. If the auto-drawn boundaries were not adequate, they were manually fixed by an expert operator and then used for the remaining acquisitions.

Inherent phase shift due to eddy currents and transient effects were removed by selecting four regions in the stationary soft-tissue mimicking liquid, which were used as phase reference for no-flow areas (NFAs).

Since $\mathbf{G}(\mathrm{t})$ and, hence, $\mathbf{m}_{\mathbf{1}}$ were perpendicular to the imaged slice, the flow rates were derived by integrating the velocity estimates on the pipe cross sections.

\section{Results}

Since the pipes were connected in series, we expected the same flow volumes per pulse period in the odd-labeled pipes (contoured by red ROIs in Fig. 2) and opposite values in the even-labeled pipes (contoured by purple ROIs in Fig. 2). No significant mismatch was actually found in the magnitude of the measured flows (Wilcoxon signed-rank test $p$-value of .950), thus excluding apparent inaccuracies in the pipe contours or in the choice of the NFAs.

\subsection{Constant flow}

The frame-wise analysis of the percentage deviation of the measured flows in the first pipe (L-1 A in Fig. 2) with respect to the values set through the calibrated pump showed a good consistency of the system (Fig. 3). This is confirmed by the mismatches averaged over the acquisition period (Table 4), which are of the order of few percentage points, except for low flow $(5 \mathrm{ml} / \mathrm{s})$ measured with a high VENC value $(75 \mathrm{~cm} / \mathrm{s})$.

Among all the performed tests, the optimum configuration is with the calibrated pump set at $10 \mathrm{ml} / \mathrm{s}$ and the VENC at 50 $\mathrm{cm} / \mathrm{s}$. We performed the pipe-by-pipe measurement of net flow over one period in such configuration. As shown in
Fig. 2 Example of magnitude (left) and phase (right) images of the phantom. Superimposition of the regions of interest of the pipes (ROIs, circles) and no flow area regions (NFAs, squares) are also reported
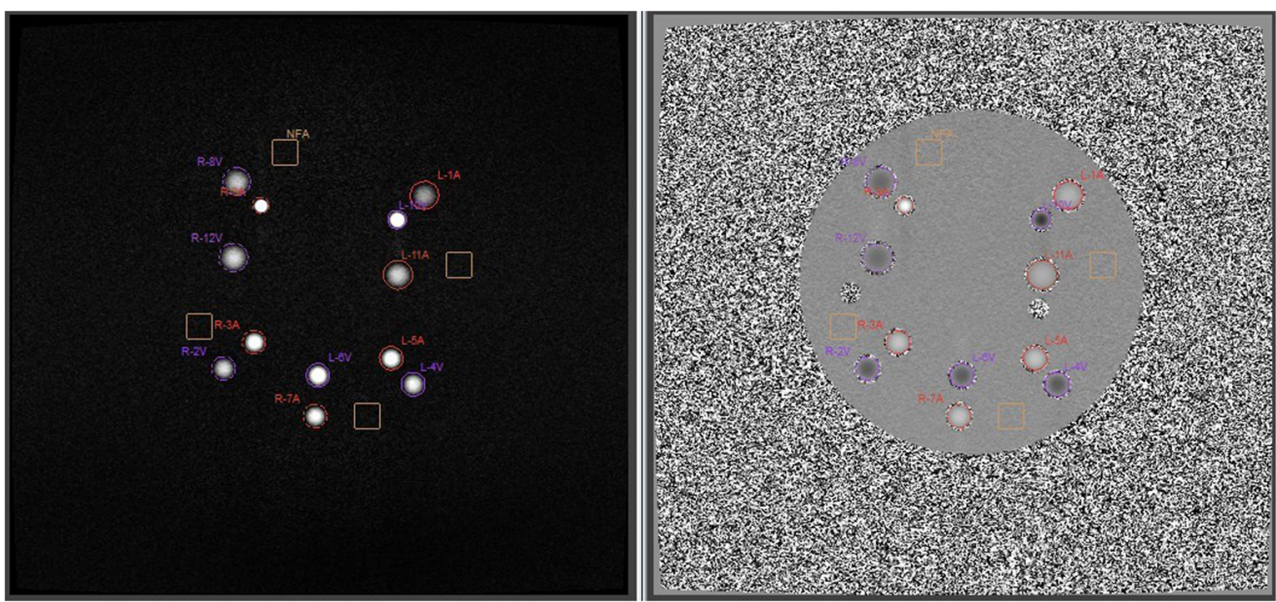
Table 3 List of symbols used in the equations

\begin{tabular}{ll}
\hline Symbol & Description \\
\hline$x(t)$ & Fluid element position \\
$G(t)$ & Flow-encoding gradient pulse \\
$T_{E}$ & Echo time \\
$\gamma$ & Gyromagnetic ratio of the imaged nuclear species \\
$\phi$ & Accumulated phase \\
$m_{n}$ & n-th moment of the flow-encoding gradient pulse \\
$\phi_{0}$ & Phase not related to flow \\
$V E N C$ & Velocity-encoding parameter \\
\hline
\end{tabular}

Fig. 4, the percentage variation from the exact value is negligible in pipe 1, and in general less than 5\%, except for pipes 9 and 10, in which some aliasing occurs (Fig. 5).

\subsection{Pulsatile flow: sine flow}

The adjusted $R^{2}$ of the linear regressions concerning the frame-wise expected flow rates vs the measured ones was plotted for sinusoidal flows grouped for frequency $(1 \mathrm{~Hz}$, Fig. 6, left) and amplitude (10 ml/s, Fig. 6, right).

An optimal match between expected and measured flows is found for large wave amplitude $(\mathrm{Q} \geq 10 \mathrm{ml} / \mathrm{s})$ or low frequency $(\mathrm{f} \leq 1 \mathrm{~Hz})$, with mean adjusted $R^{2}=0.98 \pm 0.01$. Overall, the average adjusted $R^{2}$ is $0.96 \pm 0.02$, which shows that the phantom is able to properly carry the expected sine waveform through all pipes in the analyzed range, almost regardless of the chosen frequency and amplitude. Few exceptions are found for low flow $(\mathrm{Q}=5 \mathrm{ml} / \mathrm{s}$ ) and high frequency ( $\mathrm{f}=2$ $\mathrm{Hz}$ ): in the middle-far part of the phantom (pipes 7 and 8 in Fig. 6, left, and pipes 5, 8, and 11 in Fig. 6, right), the adjusted $R^{2}$ value drops below 0.90 .

The frequency of the fitted measurements for sinusoidal flows at $\mathrm{f}=1 \mathrm{~Hz}$ (Fig. 7, left) and $\mathrm{Q}=10 \mathrm{ml} / \mathrm{s}$ (Fig. 7, right) confirms that the selected frequency is properly measured for any tested flow (with mismatches of few percent) up to an expected frequency of $\approx 1.5 \mathrm{~Hz}$. Above this threshold, large mismatches may occur between the selected frequency and the measured one. On the other hand, a clear trend can be observed between the phase of the fitted measurements and

Table 4 Percentage error averaged over the acquisition period of $1 \mathrm{~s}$ for continuous flows

\begin{tabular}{lll}
\hline & \multicolumn{2}{l}{ VENC $[\mathrm{cm} / \mathrm{s}]$} \\
\hline Flow $[\mathrm{ml} / \mathrm{s}]$ & 50 & 75 \\
5 & $-3.0 \pm 1.0$ & $-10.4 \pm 0.4$ \\
10 & $-0.1 \pm 0.7$ & $-1.0 \pm 1.0$ \\
\hline
\end{tabular}

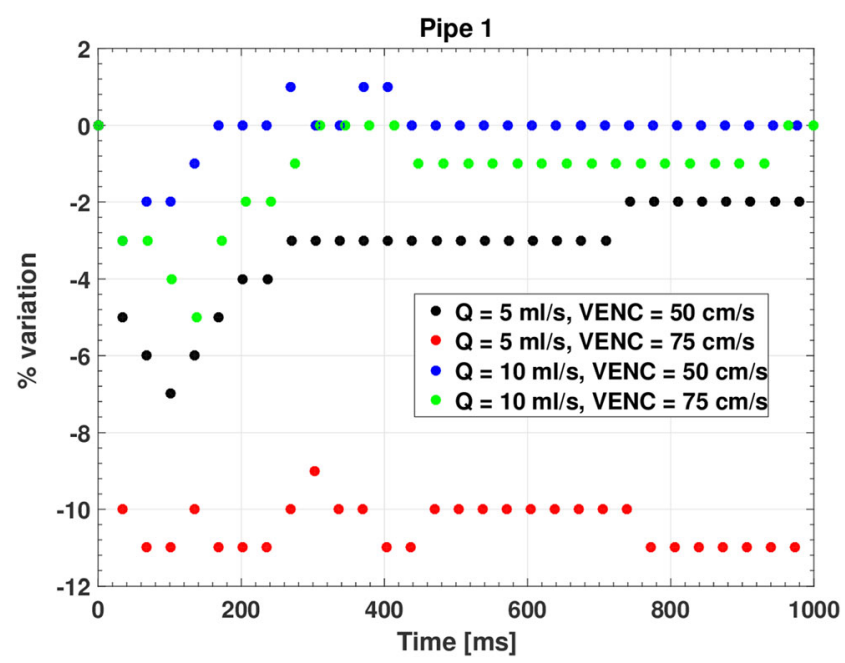

Fig. 3 Frame-by-frame measurement of continuous flows in the first pipe (L-1 A in Fig. 2)

the pipe number (Fig. 8, left (mean Spearman $\rho^{2}=0.99 \pm 0.02$, $p$-value $<.001)$ and Fig. 8 , right (mean Spearman $\rho^{2}=0.99 \pm$ $0.01, p$-value $<.001)$ ). Besides, the slope is proportional (mean Spearman $\rho^{2}=0.93, p$-value $<.001$ ) to the selected frequency of the waveform (Fig. 8 , right). This behavior is expected since the present configuration of the phantom includes some compliant tubes connecting the glass pipes that limit the phase velocity of the waves.

We also normalized the amplitudes of the fitted measurements to the expected values at the exit of the pumping system for sinusoidal flows at $\mathrm{f}=1 \mathrm{~Hz}$ (Fig. 9, left) and $\mathrm{Q}=10 \mathrm{ml} / \mathrm{s}$ (Fig. 9, right). The plots show that the normalized amplitudes decrease at increasing distance from the pump (mean Spearman $\rho^{2}=0.99 \pm 0.01, p$-value $<.001$ in Fig. 9 , left, and mean Spearman $\rho^{2}=0.63 \pm 0.37, p$-values ranging from $<$ .001 to .344 in Fig. 9, right). Also, the measured normalized amplitude is larger for high amplitude and low-frequency

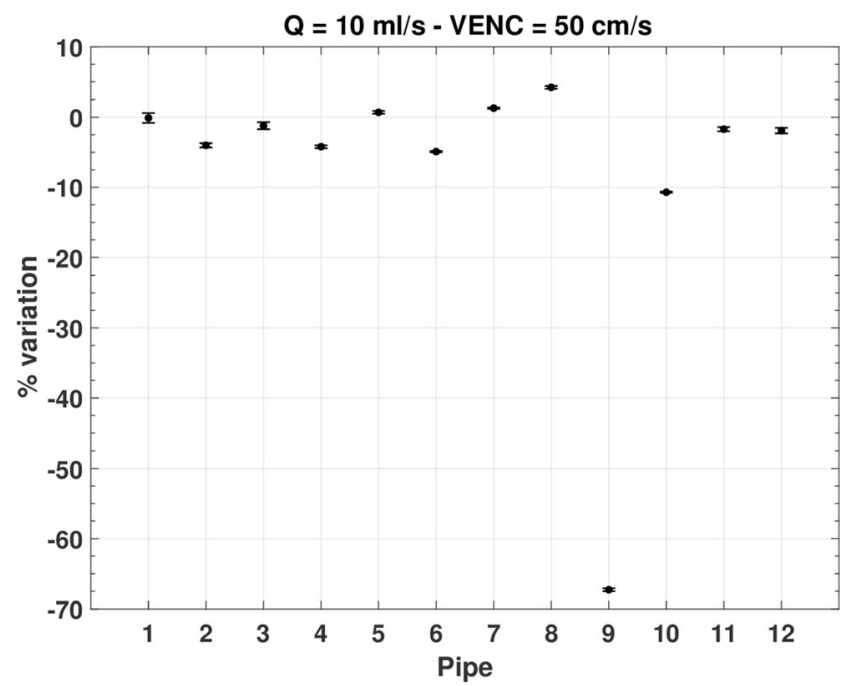

Fig. 4 Pipe-by-pipe measurement of net flow over one period (30 frames, step size $33.6 \mathrm{~ms}$ ) 


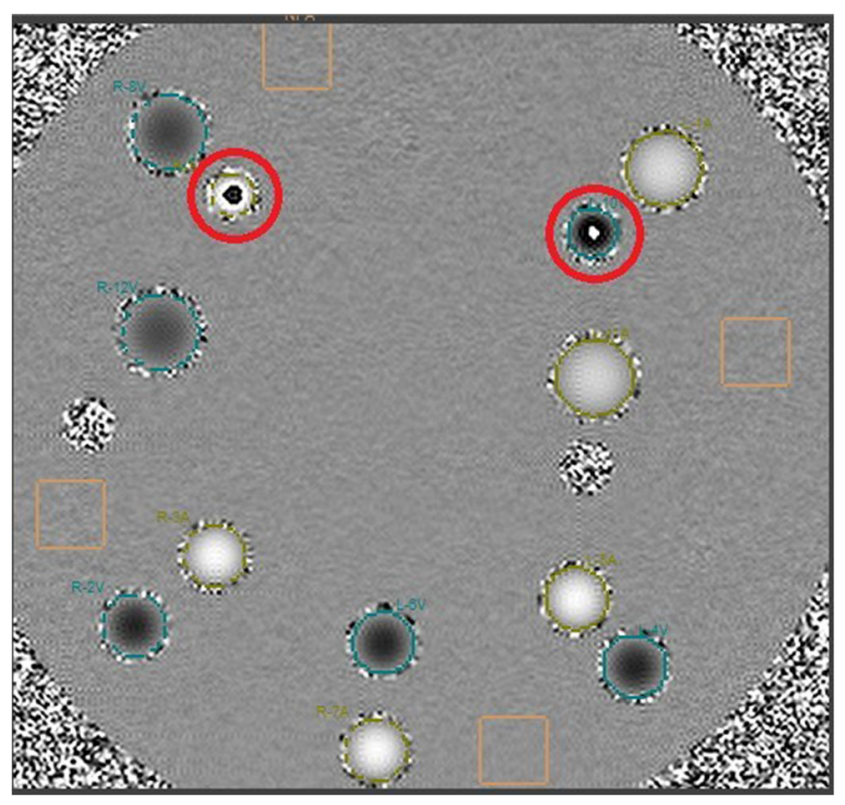

Fig. 5 MR phase image of the phantom. Pipe 9 (left red circle) and 10 (right red circle) suffer aliasing at the level of longitudinal axis

sinusoidal flows. Similarly to what happens in the phase propagation, this is due to the compliant tubes that dump the selected oscillations.

\subsection{Pulsatile flow: physiologic flow}

Similar transmission patterns of the selected waveform are found when a physiologic carotid flow [33] is mimicked with the pumping system (Fig. 10). In particular, the selected carotid flow is properly recorded by the MRI scan in all glass pipes. However, similar to the sine flows, the peaks of the measured waveforms decrease in amplitude and shift in phase as the pipe number increases.

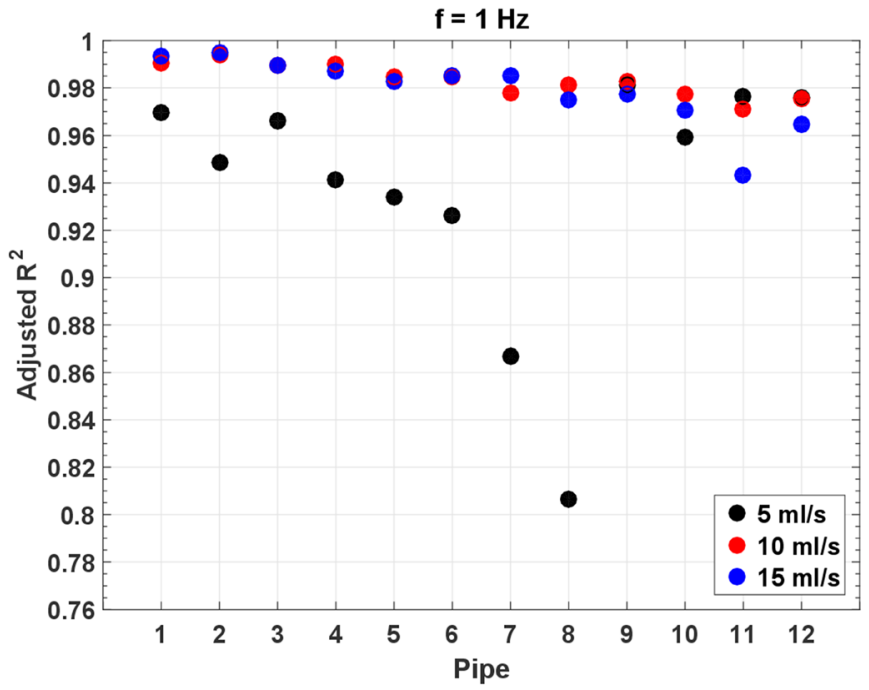

\section{Conclusion}

In the present study, we have shown the qualitative and quantitative robustness of the NO-HYPE phantom, in compliance with the specific requested design criteria [2].

The use of quantitative MR techniques is mandatory to increase the effectiveness of qualitative MRI in medicine $[34,35]$. Nevertheless, quantitative techniques strongly rely on the MRI scanner performances, thus the need of standard calibration objects [2]. The evaluation of an MRI protocol for measuring blood flow is an open and active field of research, and although many advances in MRI for flow quantification have been reached, there is still need for calibration tools [36, 37]. Indeed, some groups validated the flow patterns measured by high-resolution, time-resolved, three-dimensional PC-MRI in a real size intracranial aneurysm phantom [38], while others used a phantom to reveal that, in case of stenosis, the most accurate measures of flow by PC-MRI are found at the narrowest vessel cross section [39]. Nonetheless, it is worth to mention that these are examples of phantoms for PC-MRI flow assessment tailored on specific conditions, which cannot therefore provide an accurate and reliable estimation of the basic performances of the scanners.

Results from Fig. 6 to Fig. 7 demonstrate that the NOHYPE phantom proposed in this study is a valid tool for the analysis of any baseline offset error, which adds an unknown offset to the measured velocities. In fact, for accurate flow measurements, this offset must be shown negligible or corrected [40]. The reliability of the NO-HYPE phantom guarantees an accurate detection of this kind of error sources. In general, comparison of the reported results against literature $[2,41]$ demonstrates that the phantom is a robust standardized test object useful for the evaluation of MR flow measurements.

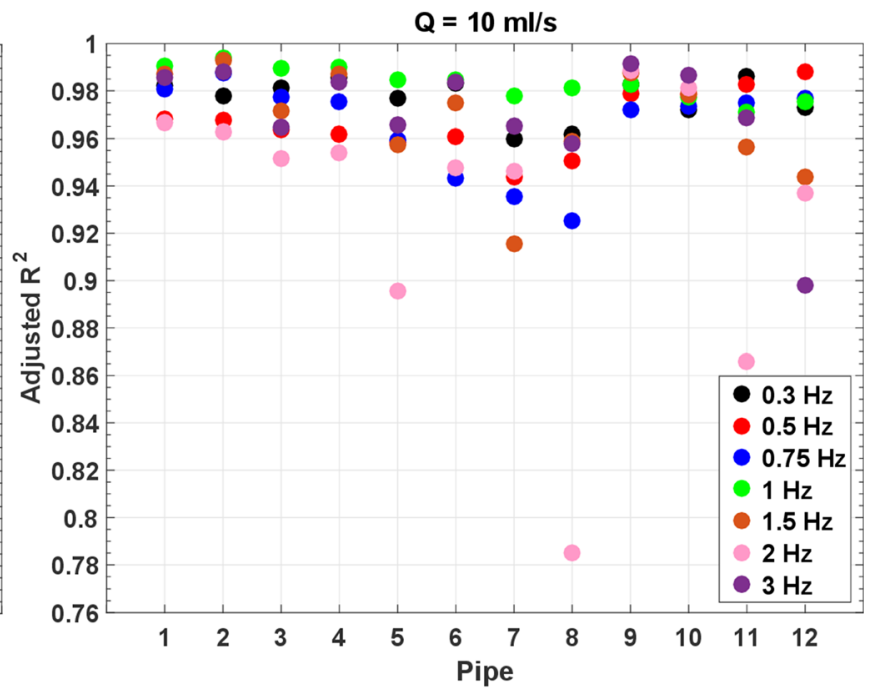

Fig. 6 Adjusted $R^{2}$ for sinusoidal flows at $\mathrm{f}=1 \mathrm{~Hz}$ (left) and $\mathrm{Q}=10 \mathrm{ml} / \mathrm{s}$ (right) 

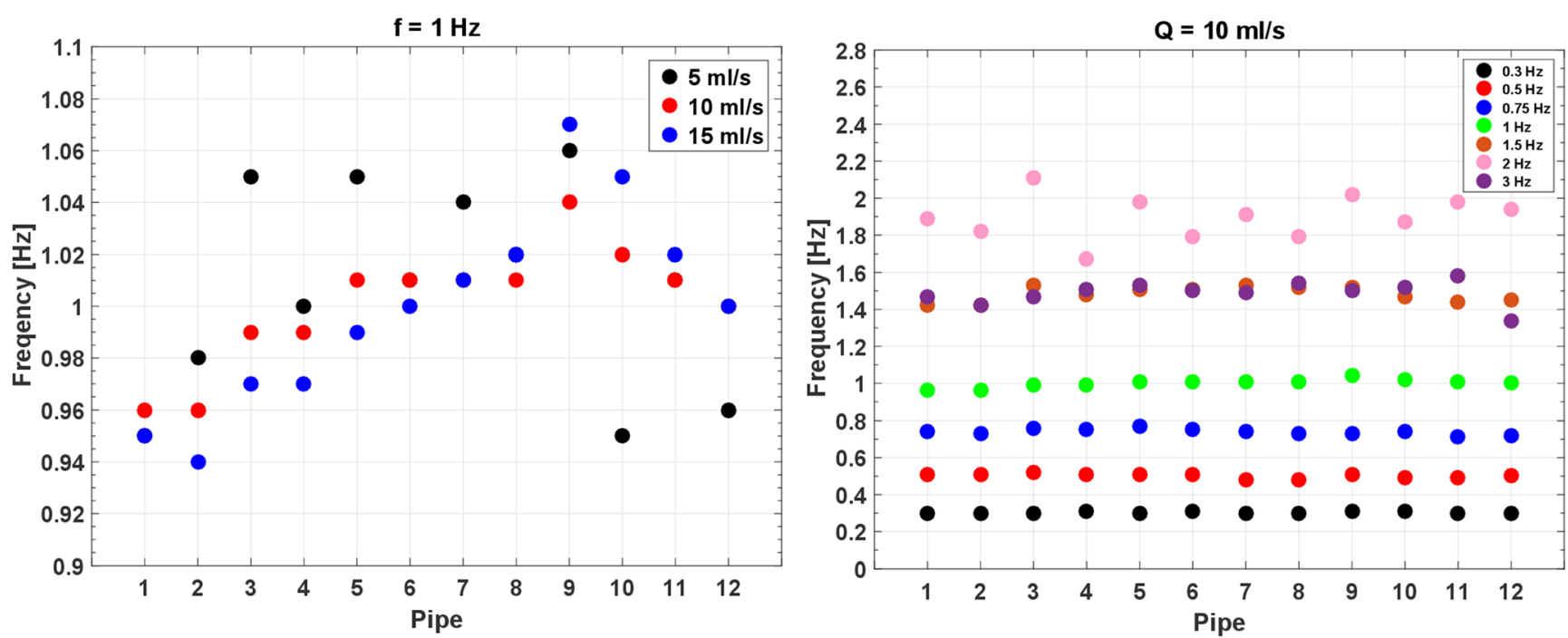

Fig. 7 Measured frequency for sinusoidal flows at $\mathrm{f}=1 \mathrm{~Hz}$ (left) and $\mathrm{Q}=10 \mathrm{ml} / \mathrm{s}$ (right)

Application-specific phantoms are largely used in clinical practice. However, they could be difficult to interface with different settings. For quantitative imaging to reach its full potential, it is necessary to analyze measurements across systems [2]. Clinical use of quantitative imaging can be facilitated through adoption and use of a standard system phantom, a calibration/standard reference object, to assess the performance of an MRI machine, or to compare and evaluate new systems for vascular monitoring $[42,43]$. The presented phantom ensures quantitative MR measurement comparable over time. Moreover, its simple structure and interface with the clinical system allows it to be easily used in every MR site and with every MR machine avoiding complicated pre-settings. To date, the most extensive studies about PC-MRI have used static tissue phantoms [2]. Static tissue phantoms can be used to study phase offset errors that have large effects on the accuracy of spatially and temporally integrated phase-contrast flow measurements. The most comprehensive phantom consisted of 10- to 15-1 tanks of aqueous gelatin solution, which were doped with $5 \mathrm{mmol} / \mathrm{l}$ of gadoliniumdiethylenetriamine pentaacetic acid to facilitate the measurement of small background phase offsets [44]. The design of the NO-HYPE phantom is promising for studies about phase offset errors with a significant reduction of the phantom volume. There is also the need for a robust, dynamic phantom to replicate spatially and temporally varying velocities across a large range of magnitudes. In single-center, in-house studies, dynamic fluid phantoms were used to replicate pulsatile flows [45]. From this point of view, the benefit of the proposed phantom relies in the fact that it has 12 pipes that allow to check flow differences in time and space, giving room to studies of up to 12 different simultaneous pulsations. There are also studies about numerical phantoms to mimick stenotic geometries [46]. From this point of view, the benefit of the
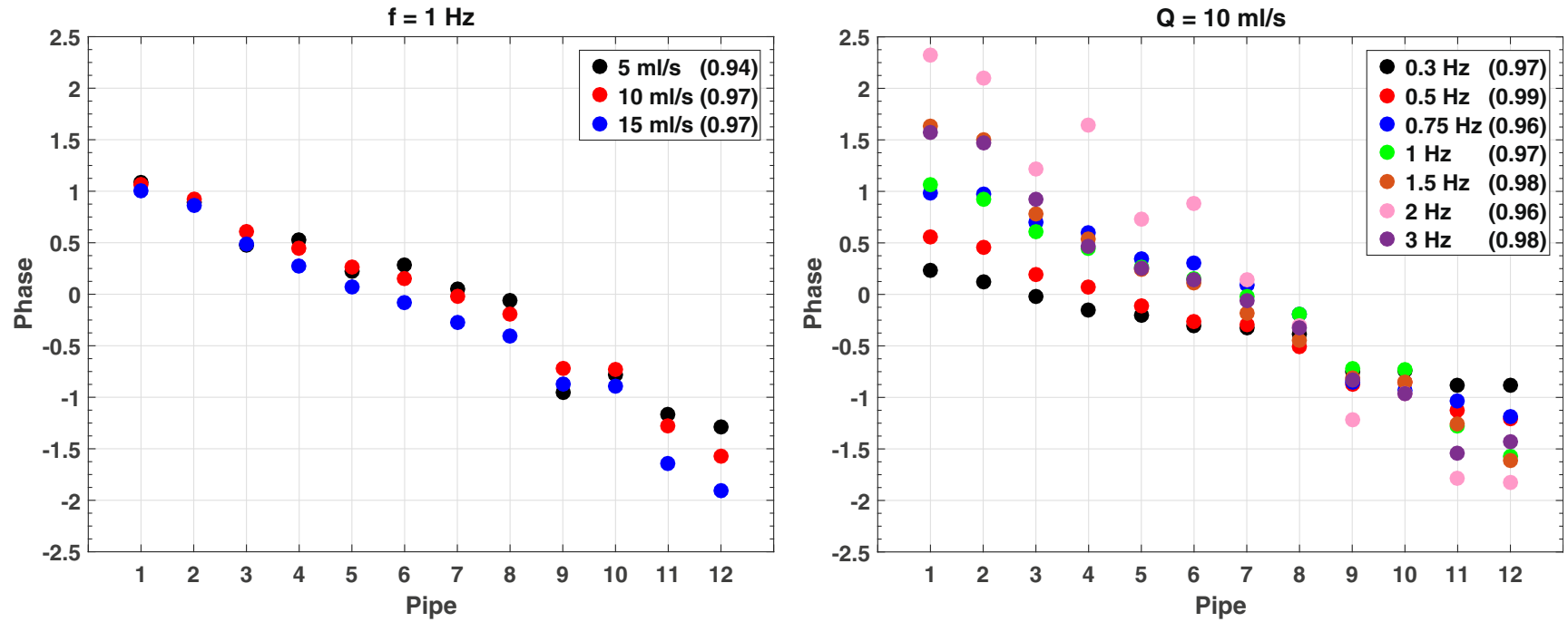

Fig. 8 Measured phase for sinusoidal flows at $\mathrm{f}=1 \mathrm{~Hz}$ (left) and $\mathrm{Q}=10 \mathrm{ml} / \mathrm{s}$ (right). Adjusted $R^{2}$ for every flow is also reported 

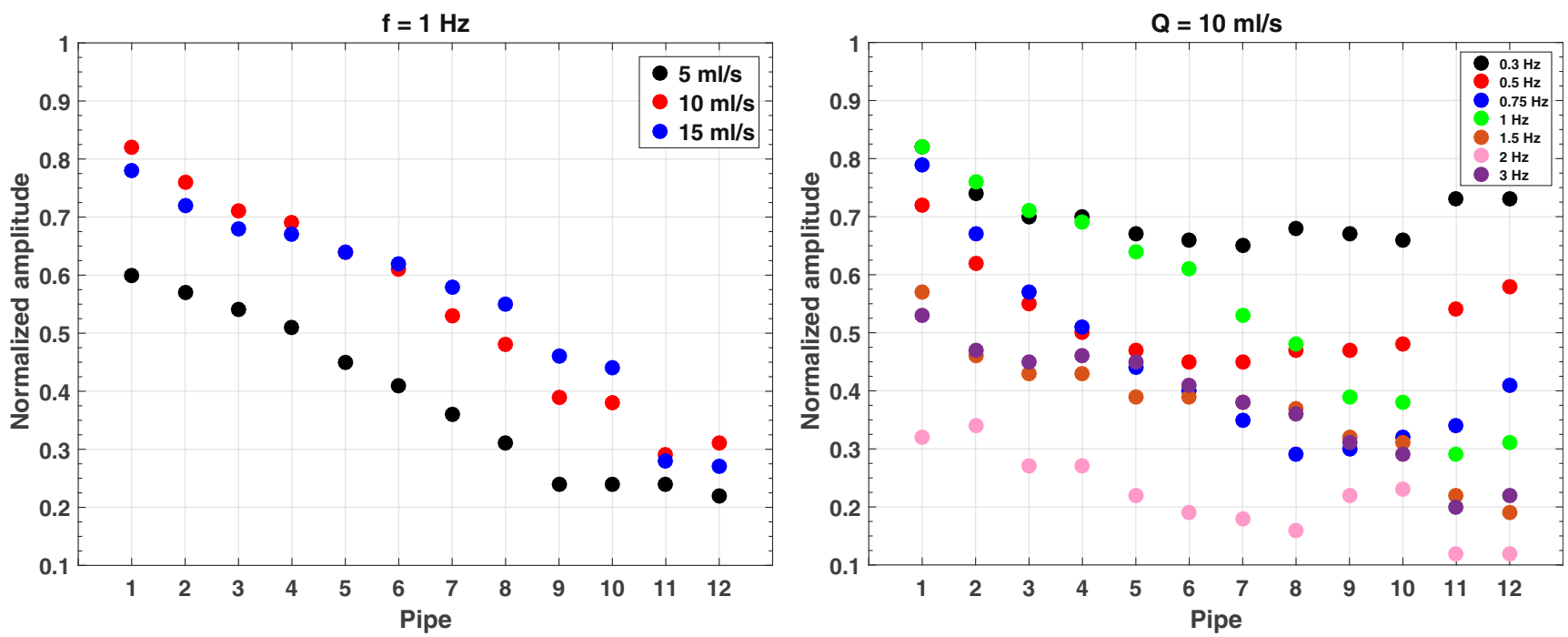

Fig. 9 Normalized amplitude for sinusoidal flows at $\mathrm{f}=1 \mathrm{~Hz}$ (left) and $\mathrm{Q}=10 \mathrm{ml} / \mathrm{s}$ (right)

proposed phantom configuration relies in the fact that it potentially allows for simultaneous in vitro simulations of stenosis in different vessels, and/or patient specific situations, avoiding numerical simulations.

Merits of the proposed design include the compatibility with a large fraction of coil geometry, the robustness and the long-term stability of the materials, and, last but not least, the production cost commensurate with existing phantoms. Beside calibration purposes, the proposed design of phantom can be used to assess the feasibility of an MR scan to evaluate a specific biomarker and to allow for periodic quality assessment tests [45-48]. Given these features, the NO-HYPE phantom could be therefore used not only for single-institution purposes, but also in the framework of multicenter clinical trials, particularly in the first steps of the study (namely, the protocol standardization and image quality assessment).

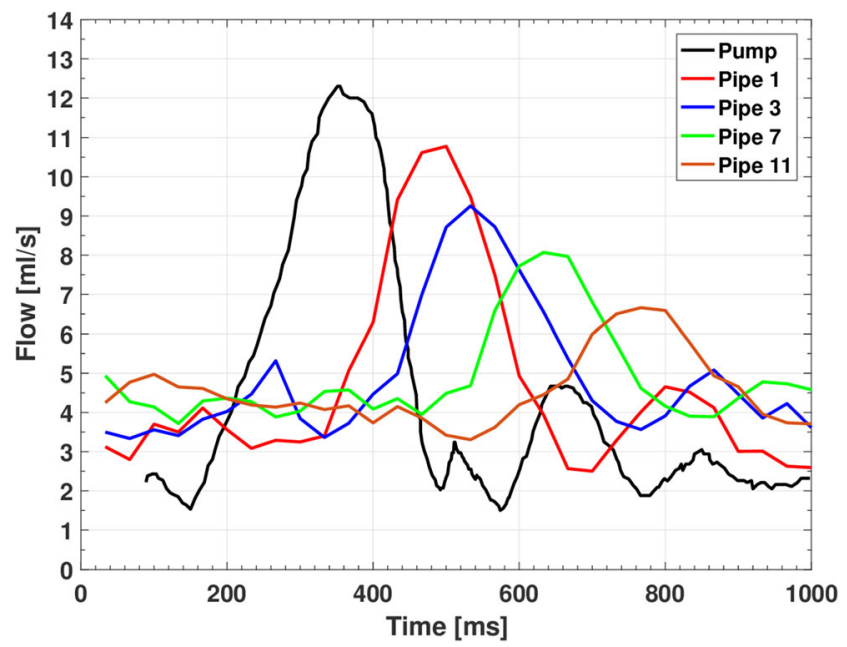

\subsection{Design constraints and future modifications}

It should be acknowledged that the present study shows a prototypical object, which obviously suffers some drawbacks. In particular, the compliance of the connections between pipes leads to an undesired dumping of the propagated waveform. This could induce a detrimental decline of the gold standard reliability of the expected flow in the farthest pipes. Also, the relaxation properties of the BMF can be further refined in order to provide phantom images comparable to clinical acquisitions. Despite these limitations, the proposed NO-

HYPE phantom offers a straightforward and cost effective approach to test the quality of PC acquisitions in clinical scanners, with a valuable impact in the estimation of flows by means of MRI. Furthermore, since the NO-HYPE phantom flows have been validated against PC-MRI measurements, the designed tool could be used for ultrasound imaging assessment by replacing the current BMF with a fluid enhancing the Doppler response [31].

Abbreviations BMF, Blood-mimicking fluid; DICOM, Digital Imaging and Communications in Medicine MRI Magnetic resonance imaging; NFA, No-flow area; PC, Phase contrast; PVC, PolyVinyl chloride; QIBA, Quantitative Imaging Biomarkers Alliance; ROI, Region of interest; RSNA, Radiological Society of North America; SNR, Signal-tonoise ratio; VENC, Velocity encoding; WSS, Wall shear stress

Author contribution G.G., M.G., A.T., P.Z., and G.P. conceived the experiment and designed the phantom. G.G., S.C., M.G., A.T., E.T., and G.P. performed the measurements. G.G. and G.P. performed data analysis and wrote the initial draft of the manuscript. All authors contributed to editing and improving the manuscript.

Fig. 10 Measured physiologic carotid flow 
Funding Open access funding provided by Università degli Studi di Ferrara within the CRUI-CARE Agreement. This study was partially supported by the Italian Ministry of Education, University and Research (MIUR Programme PRIN 2010-2011) Grant No. 2010XE5L2R.

\section{Declarations}

Competing interests The authors declare no competing interests.

Open Access This article is licensed under a Creative Commons Attribution 4.0 International License, which permits use, sharing, adaptation, distribution and reproduction in any medium or format, as long as you give appropriate credit to the original author(s) and the source, provide a link to the Creative Commons licence, and indicate if changes were made. The images or other third party material in this article are included in the article's Creative Commons licence, unless indicated otherwise in a credit line to the material. If material is not included in the article's Creative Commons licence and your intended use is not permitted by statutory regulation or exceeds the permitted use, you will need to obtain permission directly from the copyright holder. To view a copy of this licence, visit http://creativecommons.org/licenses/by/4.0/.

\section{References}

1. Paganelli C, Summers P, Gianoli C, Bellomi M, Baroni G, Riboldi M (2017) A tool for validating MRI-guided strategies: a digital breathing CT/MRI phantom of the abdominal site. Med Biol Eng Comput 55(11):2001-2014. Available from:. https://doi.org/10. 1007/s11517-017-1646-6

2. Keenan KE, Ainslie M, Barker AJ, Boss MA, Cecil KM, Charles C et al (2018) Quantitative magnetic resonance imaging phantoms: a review and the need for a system phantom. Magn Reson Med 79(1): 48-61. Available from:. https://doi.org/10.1002/mrm.26982

3. Moran PR (1982) A flow velocity zeugmatographic interlace for NMR imaging in humans. Magn Reson Imaging 1(4):197-203. Available from:. https://doi.org/10.1016/0730-725x(82)90170-9

4. Pelc NJ, Herfkens RJ, Shimakawa A, Enzmann DR (1991) Phase contrast cine magnetic resonance imaging. Magn Reson Q 7(4): 229-254

5. Pope JM, Yao S (1993) Quantitative NMR imaging of flow. Concepts Magn Reson 5(4):281-302. Available from:. https://doi. org/10.1002/cmr.s1820050402

6. Firmin DN, Nayler GL, Kilner PJ, Longmore DB (1990) The application of phase shifts in NMR for flow measurement. Magn Reson Med 14(2):230-241. Available from:. https://doi.org/10. 1002/mrm.1910140209

7. Ku DN, Biancheri CL, Pettigrew RI, Peifer JW, Markou CP, Engels H (1990) Evaluation of magnetic resonance velocimetry for steady flow. J Biomech Eng 112(4):464 472. Available from:. https://doi. org $/ 10.1115 / 1.2891212$

8. Adib MAHM, Ii S, Watanabe Y, Wada S (2017) Minimizing the blood velocity differences between phase-contrast magnetic resonance imaging and computational fluid dynamics simulation in cerebral arteries and aneurysms. Med Biol Eng Comput 55(9):1605, 1619. Available from. https://doi.org/10.1007/s11517-017$1617-\mathrm{y}$

9. Gatehouse PD, Keegan J, Crowe LA, Masood S, Mohiaddin RH, Kreitner KF et al (2005) Applications of phase-contrast flow and velocity imaging in cardiovascular MRI. Eur Radiol 15(10):2172 2184. Available from:. https://doi.org/10.1007/s00330-005-2829-3

10. Zhao M, Charbel FT, Alperin N, Loth F, Clark ME (2000) Improved phase-contrast flow quantification by three-dimensional vessel localization. Magn Reson Imaging 18(6):697-706. Available from:. https://doi.org/10.1016/s0730-725x(00)00157-0

11. Yang GZ, Kilner PJ, Wood NB, Underwood SR, Firmin DN (1996) Computation of flow pressure fields from magnetic resonance velocity mapping. Magn Reson Med 36(4):520-526. Available from:. https://doi.org/10.1002/mrm.1910360404

12. Man LC, Pauly JM, Nishimura DG, Macovski A (1999) Non- subtractive spiral phase contrast velocity imaging. Magn Reson Med 42(4):704-713. Available from:. https://doi.org/10.1002/(SICI) 1522-2594(199910)42:4<704::AID-MRM12>3.0.CO;2-M

13. Yeh HH, Rabkin SW, Grecov D (2018) Hemodynamic assessments of the ascending thoracic aortic aneurysm using fluid-structure interaction approach. Med Biol Eng Comput 56(3):435-451. Available from:. https://doi.org/10.1007/s11517-017-1693-Z

14. Canstein C, Cachot P, Faust A, Stalder AF, Bock J, Frydrychowicz A et al (2008) 3D MR flow analysis in realistic rapid-prototyping model systems of the thoracic aorta: comparison with in vivo data and computational fluid dynamics in identical vessel geometries. Magn Reson Med 59(3):535-546. Available from:. https://doi.org/ $10.1002 / \mathrm{mrm} .21331$

15. Stalder AF, Russe MF, Frydrychowicz A, Bock J, Hennig J, Markl M (2008) Quantitative 2D and 3D phase contrast MRI: optimized analysis of blood flow and vessel wall parameters. Magn Reson Med 60(5):1218-1231. Available from:. https://doi.org/10.1002/ mrm. 21778

16. Cocozza S, Canna A, Lanzillo R, Russo C, Postiglione E, Liuzzi R et al (2016) Lack of correlation between extracranial venous abnormalities and multiple sclerosis: a quantitative MRI study. Br J Radiol 89(1064):20160321. Available from:. https://doi.org/10. 1259/bjr.20160321

17. Boussel L, Rayz V, Martin A, Acevedo-Bolton G, Lawton MT, Higashida R et al (2009) Phase-contrast magnetic resonance imaging measurements in intracranial aneurysms in vivo of flow patterns, velocity fields, and wall shear stress: comparison with computational fluid dynamics. Magn Reson Med 61(2):409-417. Available from:. https://doi.org/10.1002/mrm.21861

18. Radiological Society of North America. Quantitative imaging biomarkers alliance. Available from: https:/www.rsna.org/en/research/quantitative- imaging-biomarkers-alliance.

19. Firbank MJ, Harrison RM, Williams ED, Coulthard A (2000) Quality assurance for MRI: practical experience. Br J Radiol 73(868):376-383. Available from:. https://doi.org/10.1259/bjr.73. 868.10844863

20. Jackson EF, Bronskill MJ, Drost DJ, Och J, Pooley RA, Sobol WT, et al (2010) Acceptance testing and quality assurance procedures for magnetic resonance imaging facilities; AAPM Report 100. American Association of Physicists in Medicine, College Park, MD. Available from: https://doi.org/10.37206/101

21. Lerski RA, de Certaines JD (1993) Performance assessment and quality control in MRI by Eurospin test objects and protocols. Magn Reson Imaging 11(6):817-833. Available from:. https://doi. org/10.1016/0730-725x(93)90199-n

22. Price RR, Axel L, Morgan T, Newman R, Perman W, Schneiders N et al (1990) Quality assurance methods and phantoms for magnetic resonance imaging: report of AAPM nuclear magnetic resonance Task Group No. 1. Med Phys 17(2):287-295. Available from:. https://doi.org/10.1118/1.596566

23. Shelley Medical Imaging Technologies. Compuflow $1000 \mathrm{MR}$, pro- grammable physiological flow pump \& accessories. Available from: https://www.simutec.com/Products/ppfp.html

24. Holdsworth DW, Rickey DW, Drangova M, Miller DJ, Fenster A (1991) Computer-controlled positive displacement pump for physiological flow simulation. Med Biol Eng Comput 29(6):565-570. Available from. https://doi.org/10.1007/BF02446086

25. Blechinger JC, Madsen EL, Frank GR (1988) Tissue-mimicking gelatin- agar gels for use in magnetic resonance imaging phantoms. 
Med Phys 15(4):629-636. Available from. https://doi.org/10.1118/ 1.596219

26. D'Souza WD, Madsen EL, Unal O, Vigen KK, Frank GR, Thomadsen BR (2001) Tissue mimicking materials for a multiimaging modality prostate phantom. Med Phys 28(4):688-700. Available from. https://doi.org/10.1118/1.1354998

27. Madsen EL, D'Souza WD, Frank GR. Multi-imaging modality tissue mimicking materials for imaging phantoms. Available from: https://www.patents.google.com/patent/US6635486B2/en

28. McDonald M, Lochhead S, Chopra R, Bronskill MJ (2004) Multimodality tissue-mimicking phantom for thermal therapy. Phys Med Biol 49(13):2767-2778. Available from. https://doi.org/10.1088/ 0031-9155/49/13/001

29. Yuan Y, Wyatt C, Maccarini P, Stauffer P, Craciunescu O, Macfall $\mathrm{J}$ et al (2012) A heterogeneous human tissue mimicking phantom for RF heating and MRI thermal monitoring verification. Phys Med Biol 57(7):2021-2037. Available from. https://doi.org/10.1088/ $0031-9155 / 57 / 7 / 2021$

30. Meier D, Maier S, Bösiger P (1988) Quantitative flow measurements on phantoms and on blood vessels with MR. Magn Reson Med 8(1):25-34. Available from. https://doi.org/10.1002/mrm. 1910080104

31. Shelley Medical Imaging Technologies. Shelley's blood mimicking fluids datasheet. Available from: http://www.simutec.com/ Docs/BloodMimickingFluids.pdf

32. Spintech. From image to insight. Available from: https://www. spintechimaging.com/

33. Stankevich Y, Starokozheva M, Bogomyakova O, Shraybman L, Tulupov A; (2015). Quantification of the arterial blood flow through the internal carotid arteries at patients with chronic cerebral ischemia by MRI. In: ECR 2015. Corpus ID: 78710123.

34. Zhu H, He G, Wang Z (2018) Patch-based local learning method for cerebral blood flow quantification with arterial spin-labeling MRI. Med Biol Eng Comput 56(6):951-956. Available from:. https://doi.org/10.1007/s11517-017-1735-6

35. Brunner G, Bismuth J, Nambi V, Ballantyne CM, Taylor AA, Lumsden AB et al (2016) Calf muscle perfusion as measured with magnetic resonance imaging to assess peripheral arterial disease. Med Biol Eng Comput 54(11):1667-1681. Available from. https://doi.org/10.1007/s11517-016-1457-1

36. Bakker CJ, Hoogeveen RM, Viergever MA (1999) Construction of a protocol for measuring blood flow by two-dimensional phasecontrast MRA. J Magn Reson Imaging 9(1):119-127. Available from. https://doi.org/10.1002/(sici)1522-2586(199901)9:1<119:: aid-jmri16 $>3.0 . c 0 ; 2-\mathrm{f}$

37. Bidhult SL, Carlsson M, Steding-Ehrenborg K, Arheden H, Heiberg E (2014) A new method for vessel segmentation based on a priori input from medical expertise in cine phase-contrast magnetic resonance imaging. J Cardiovasc Magn Reson 16(1): P355. Available from. https://doi.org/10.1186/1532-429X-16-S1P355

38. van Ooij P, Guédon A, Poelma C, Schneiders J, Rutten MCM, Marquering HA et al (2012) Complex flow patterns in a real-size intracranial aneurysm phantom: phase contrast MRI compared with particle image velocimetry and computational fluid dynamics. NMR Biomed 25(1):14-26. Available from. https://doi.org/10. 1002/nbm.1706

39. Khodarahmi I, Shakeri M, Kotys-Traughber M, Fischer S, Sharp MK, Amini A (2012) Accuracy of flow measurement with phase contrast MRI in a stenotic phantom: where should flow be measured? J Cardiovasc Magn Reson 14(Suppl 1):P219. Available from. https://doi.org/10.1186/1532-429X-14-S1-P219

40. Gatehouse PD, Rolf MP, Bloch KM, Graves MJ, Kilner PJ, Firmin DN et al (2012) A multi-center inter-manufacturer study of the temporal stability of phase-contrast velocity mapping background offset errors. J Cardiovasc Magn Reson 14:72. Available from. https://doi.org/10.1186/1532-429X-14-72

41. Summers PE, Holdsworth DW, Nikolov HN, Rutt BK, Drangova M (2005) Multisite trial of MR flow measurement: phantom and protocol design. J Magn Reson Imaging 21(5):620-631. Available from. https://doi.org/10.1002/jmri.20311

42. Sone S, Hayase T, Funamoto K, Shirai A (2017) Photoplethysmography and ultrasonic-measurement-integrated simulation to clarify the relation between two-dimensional unsteady blood flow field and forward and backward waves in a carotid artery. Med Biol Eng Comput 55(5):719-731. Available from. https://doi.org/10.1007/s11517-016-1543-4

43. Rejmstad P, Åkesson G, Åneman O, Wårdell K (2016) A laser Doppler system for monitoring cerebral microcirculation: implementation and evaluation during neurosurgery. Med Biol Eng Comput 54(1):123-131. Available from. https://doi.org/10.1007/ s11517-015-1332-5

44. Gatehouse PD, Rolf MP, Graves MJ, Hofman MB, Totman J, Werner B et al (2010) Flow measurement by cardiovascular magnetic resonance: a multi-centre multi-vendor study of background phase offset errors that can compromise the accuracy of derived regurgitant or shunt flow measurements. J Cardiovasc Magn Reson 12(1):5. Available from. https://doi.org/10.1186/1532429X-12-5

45. Barker AJ, Lanning C, Shandas R (2010) Quantification of hemodynamic wall shear stress in patients with bicuspid aortic valve using phase-contrast MRI. Ann Biomed Eng 38(3):788-800. Available from. https://doi.org/10.1007/s10439-009-9854-3

46. Casas B, Lantz J, Dyverfeldt P, Ebbers T (2016) 4D Flow MRIbased pressure loss estimation in stenotic flows: evaluation using numerical simulations. Magn Reson Med 75(4):1808-1821. Available from. https://doi.org/10.1002/mrm.25772

47. Bosca R, Ashton EA, Zahlmann G, Jackson EF; (2012). RSNA quantitative imaging biomarker alliance (QIBA) DCE-MRI phantom: goal, design, and initial results. In: Radiological Society of North America. Scientific Assembly and Annual Meeting

48. Gunter JL, Bernstein MA, Borowski BJ, Ward CP, Britson PJ, Felmlee JP et al (2009) Measurement of MRI scanner performance with the ADNI phantom. Med Phys 36(6):2193-2205. Available from. https://doi.org/10.1118/1.3116776

Publisher's note Springer Nature remains neutral with regard to jurisdictional claims in published maps and institutional affiliations.

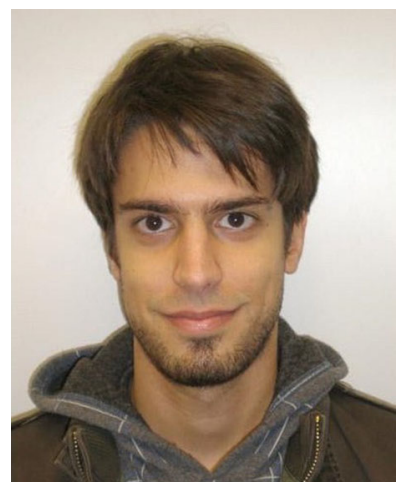

Giacomo Gadda is a research fellow at the National Institute for Nuclear Physics (INFN). His expertise covers various aspects of medical physics, from wearable sensors to non-ionizing imaging and modeling of the cardiovascular system. 


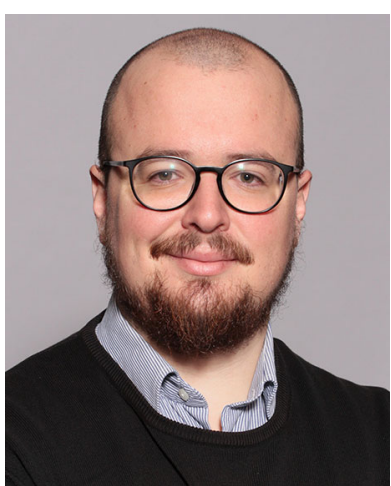

Sirio Cocozza MD, is enrolled in a $\mathrm{PhD}$ program at the University of Napoli. His research is focused on the application of advanced MRI techniques to investigate neuroinflammatory and neurodegenerative disorders.

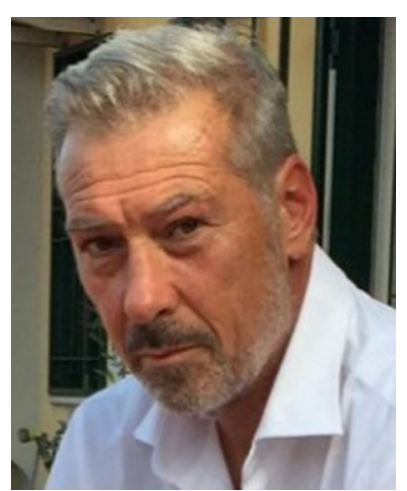

Enrico Tedeschi is an Associate Professor of Neuroradiology at the University of Napoli. His research fields mainly cover the applications of Magnetic Resonance Imaging in demyelinating diseases.

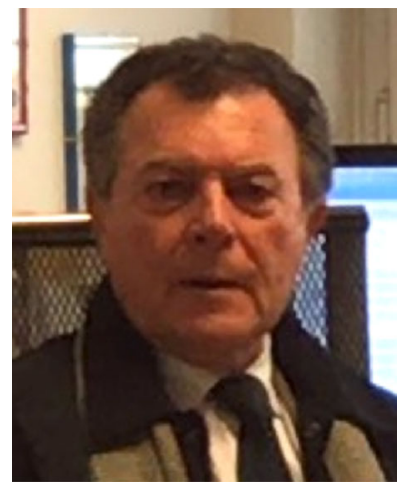

Mauro Gambaccini is a Full Professor of Medical Physics at the University of Ferrara. His expertise concerns the optimization of clinical diagnostic systems in radiology and nuclear medicine, and modeling of the cardiovascular system.
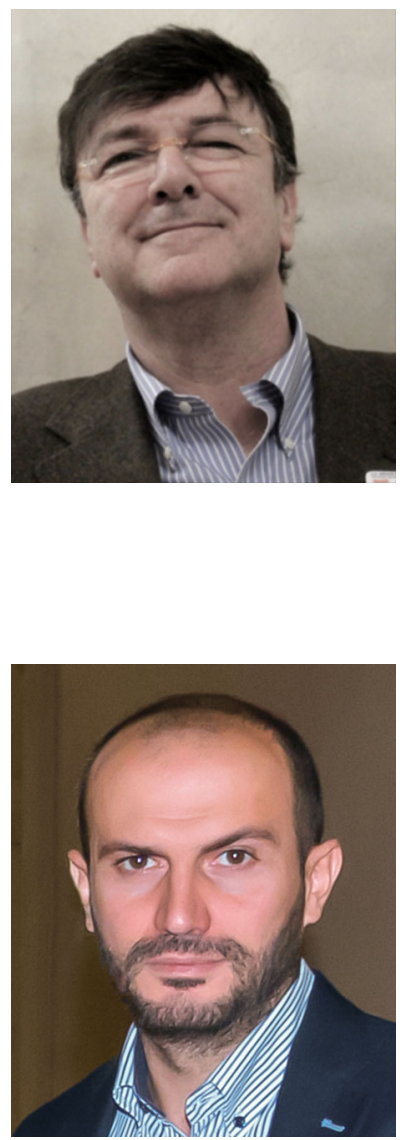

Paolo Zamboni MD, is a Full Professor of Vascular Surgery at the University of Ferrara. His expertise covers neurovascular diseases, venous and lymphatics disorders including diagnostics and hemodynamic models.

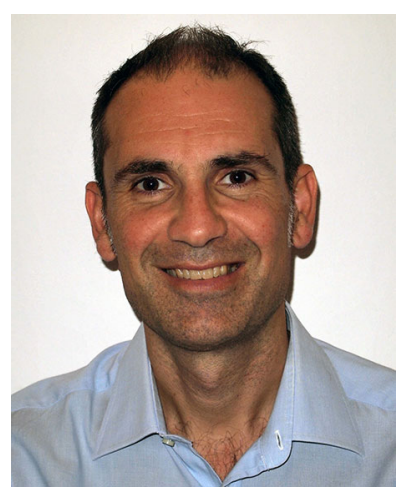

Angelo Taibi is an Associate Professor of Medical Physics at the University of Ferrara. His expertise covers various aspects of the physics of diagnostic radiology, nuclear medicine, and biophysics of blood circulation.
Giuseppe Palma is a research scientist for the Italian National Research Council. His areas of expertise include theoretical and medical physics, image processing, and computer science. 